\title{
Betreiber von Webseiten benötigen Rechtssicherheit
}

\section{Bundesgesetzgeber muss europarechtliche Verpflichtungen der „ePrivacyRichtlinie" endlich erfüllen}

Der Gesetzgeber ist verpflichtet, die EU-Richtlinie über den europäischen Kodex für die elektronische Kommunikation vom 11. Dezember 2018 (RL 2018/1972/EU) bis zum 20. Dezember 2020 umzusetzen. Die Konferenz der unabhängigen Datenschutzaufsichtsbehörden des Bundes und der Länder (DSK) fordert den Gesetzgeber auf, endlich Regelungen zu erlassen, um die ePrivacy-Richtlinie ${ }^{1}$ vollständig und im Einklang mit der DSGVO umzusetzen.

Die DSK hat in der Vergangenheit wiederholt kritisch darauf hingewiesen, dass der Gesetzgeber Art. 5 Abs. 3 ePrivacy-Richtlinie nicht oder nicht ordnungsgemäß umgesetzt hat ${ }^{2}$. Das Urteil des Bundesgerichtshofs (BGH) vom 28. Mai 2020 (I ZR 7/16 „Planet49“) verstärkt nach Auffassung der DSK den seit langem bestehenden, dringenden Handlungsbedarf.

Die DSK hat bereits im April 2018 in der Positionsbestimmung „Zur Anwendbarkeit des TMG für nichtöffentliche Stellen ab dem 25. Mai 2018“ den Standpunkt vertreten, dass die Datenschutzvorschriften des Telemediengesetzes neben der DSGVO nicht mehr anwendbar sind. Eine ausführliche Begründung zu dieser Rechtsauffassung wurde von der DSK in der Orientierungshilfe für Anbieter von Telemedien im März 2019 veröffentlicht ${ }^{3}$.

Der BGH hatte im Planet49-Verfahren einen Streit zu entscheiden, in dem das beklagte Unternehmen personenbezogene Daten über das Nutzungsverhalten von Verbrauchern mittels Cookies zu pseudonymisierten Nutzungsprofilen verarbeitete und diese für personalisierte Werbung nutzte. Nach dem Wortlaut des $\$$ 15 Abs. 3 Telemediengesetz (TMG) wäre ein solches Vorgehen dann zulässig, wenn die betroffenen Personen entsprechend informiert wurden und nicht widersprochen haben (sogenannte Widerspruchslösung). Mit Blick auf Art. 5 Abs. 3 ePrivacyRichtlinie legt der BGH $\$ 15$ Abs. 3 TMG dahingehend aus, schon in dem Fehlen einer wirksamen Einwilligung könne ein solcher Widerspruch gesehen werden, weshalb eine aktive Einwilligung

1 Richtlinie 2002/58/EG in der letzten Änderung durch die Richtlinie 2009/136/EU

2 Siehe Umlaufentschließung der Datenschutzbeauftragten des Bundes undder Länder vom 05. Februar 2015, abrufbar unter: https://www.datenschutzkonferenzonline.de/media/en/20150205_en_Entschliessung_Cookies.pdf

3 Positionsbestimmung der DSK vom 26. April 2018 „Zur Anwendbarkeit des TMG für nicht-öffentliche Stellen ab dem 25. Mai 2018“, abrufbar unter: https:// www.datenschutzkonferenzonline.de/anwendungshinweise.html), Orientierungshilfe für Anbieter von Telemedien (https://www.datenschutzkonferenz-online.de/media/oh/20190405_oh_tmg.pdf erforderlich sei. Unter Zugrundelegung dieser Auslegung von $\$$ 15 Abs. 3 TMG wendet er diese Vorschrift neben der DSGVO an. Letztlich ist der BGH der Vorabentscheidung des Europäischen Gerichtshofes gefolgt und bestätigt das grundsätzliche Erfordernis einer wirksamen Einwilligung für das Setzen von Cookies.

Schon die Tatsache, dass die DSK und der BGH bei einer sehr praxisrelevanten Rechtsfrage zwar im Ergebnis darin übereinstimmen, dass eine Verarbeitung, wie sie den Gerichten zur Entscheidung vorlag, einwilligungsbedürftig ist, jedoch bei der Herleitung dieses Ergebnisses voneinander abweichende Auffassungen vertreten, verdeutlicht das Ausmaß der Rechtsunklarheit.

Mit der Entscheidung wird die Abgrenzung der Regelungsbereiche zwischen ePrivacyRichtlinie, DSGVO und den Datenschutzvorschriften des TMG deutlich erschwert. Der BGH stellt ausdrücklich heraus, dass ePrivacy-Richtlinie und DSGVO unterschiedliche Schutzrichtungen verfolgen. Die Vorschriften in den $\$ \$ 12$ bis 15 TMG knüpfen ausdrücklich an den Begriff der Verarbeitung personenbezogener Daten an. Diese Materie ist auf europäischer Ebene weitgehend abschließend durch die DSGVO geregelt. Art. 5 Abs. 3 ePrivacy-Richtlinie hat hingegen auch Informationen ohne Personenbezug zum Regelungsgegenstand. Es bleibt daher offen, ob $\$ 15$ Abs. 3 TMG - entgegen des Wortlautes - auch dann eine Umsetzung des Art. 5 Abs. 3 ePrivacy-Richtlinie darstellen soll, wenn die Informationen, die im Endgerät eines Teilnehmers gespeichert werden oder auf die zugegriffen wird, keinen Personenbezug haben.

$\$ 15$ Abs. 3 TMG bezieht sich ausdrücklich und ausschließlich auf die Erstellung von pseudonymen Nutzungsprofilen für Zwecke der Werbung, der Marktforschung oder zur bedarfsgerechten Gestaltung der Telemedien. Die Speicherung von Informationen oder der Zugriff auf Informationen, die bereits im Endgerät eines Teilnehmers oder Nutzers gespeichert sind, kann jedoch auch zu anderen Zwecken erfolgen und ist nicht auf die in $\$$ 15 Abs. 3 TMG genannten Zwecke beschränkt.

Schließlich fordert Art. 5 Abs. 3 ePrivacy-Richtlinie grundsätzlich ohne Berücksichtigung konkreter Zwecke eine Einwilligung. Lediglich in Art. 5 Abs. 3 Satz 2 ePrivacy-Richtlinie finden sich Ausnahmen von diesem Grundsatz. Dieses RegelAusnahmePrinzip findet sich im TMG nicht wieder.

Webseitenbetreiber und andere Akteure, die ihre Dienste u. a. in Bezug auf „Cookies“ rechtskonform gestalten müssen, brauchen Rechtsklarheit. Der Gesetzgeber ist deshalb aufgefordert, bestehende Rechtsunsicherheiten umgehend durch eine klare und europarechtskonforme Gesetzgebung zu beseitigen. 